\title{
NECESIDADES DE CUALIFICACIÓN DE LOS ENFERMEROS DE SALUD DE LA FAMILIA EN CEARÁ, BRASIL
}

\author{
NECESSIDADES DE QUALIFICAQÁO DOS ENFERMEIROS DA ESTRATEGIA SAÚDE \\ DA FAMÍLIA NO CEARÁ, BRASIL
}

\author{
"Ximenes Neto, FRG., "*Ponte, MAC., " ${ }^{* * * A m a r a l, ~ M I V ., ~}{ }^{* \star *}$ Chagas, MIO., " ${ }^{* \star * * D i a s, ~}$ \\ MS de A., ${ }^{* \star * \star * C u n h a, ~ I C K O . ~}$
}

*Mestre em Saúde Pública. Docente do Curso de Enfermagem da Universidade Estadual do Vale do A caraú-UVA. **Enfermeira Obstetra, Professora do Curso de Enfermagem da UVA. ***Enfermeira Sanitarista da Secretaria da Saúde do Estado do Ceará-Brasil. ****Doutora em Enfermagem. Coordenadora do Curso de Enfermagem da UVA, Sobral, Ceará. $* * * *$ Doutora em Enfermagem. Coordenadora Adjunto do Curso de Enfermagem da UVA, Sobral-Ceará. $* * * * *$ Doutora em Saúde Pública. Professora Adjunta e Líder Grupo de Estudos e Pesquisas em Administracáo em Saúde e Gerenciamento de Enfermagem-GEPAG da Universidade Federal de Sáo Paulo-UNIFESP. Brasil.

Palabras clave: Enfermera; Cualificación Profesional; Salud de la Familia Palavras-chave: Enfermeiro; Qualificagao Profissional, Saúde da Familia

\section{RESUMEN}

El estudio tiene el objetivo de identificar las necesidades de cualificación y el perfil de los enfermeros de la Estrategia Salud de la Familia-ESF. La investigación es del tipo exploratoriodescriptivo, realizada con 36 enfermeros de las provincias de Acaraú, Coreaú, Chaval, Granja, Marco e Santana do Acaraú, que actúan en la ESF y alumnos del Curso de Especialización en la modalidad de Residencia en Salud de la Familia administrado por la Escuela de Formación en Salud de la Familia Visconde de Sabóia. Las provincias son las que forman parte del Proyecto Acciones Básicas de Salud del Ceará,- Programa de Cualificación de la Secretaria de Salud del Estado del Ceará, Brasil, en sociedad con la agencia alemana Kreditanstalt für Wiederaufdau-KfW y el Instituto Brasilero de Qualidade Nuclear-IBQN. Para la colecta de datos fueron realizados seis talleres durante el mes de julio de 2004, en las propias provincias, momento en que fue aplicado un cuestionario. Las necesidades de cualificación están centradas en acciones programáticas, relativas a las diferentes fases de la vida - infancia, adolescencia, edad adulta y vejez - como también la legislación, métodos y procedimientos comunes al quehacer del enfermero/a. Atendimos a las necesidades de una formación de los enfermeros con referencia a las concepciones de la promoción de la salud, organización del servicio, cuidado familiar en la perspectiva del concepto de territorio. 


\section{RESUMO}

0 estudo objetiva identificar as necessidades de qualificacáo e o perfil dos enfermeiros da Estrategia Saúde da Família-ESF. A pesquisa do tipo exploratório-descritivo, realizado com 36 enfermeiros dos municipios de Acaraú, Coreaú, Chaval, Granja, Marco e Santana do Acaraú, que atuam na ESF e alunos do Curso de Especializacáo na modalidade de Residencia em Saúde da Familia ministrado pela Escola de Formacáo em Saúde da Familia Visconde de Sabóia. Os municipios fazem parte do Projeto Acóes Básicas de Saúde do Ceará - Programa de Qualificacáo da Secretaria da Saúde do Estado do Ceará,Brasil, em parceria com a agencia alemá Kreditanstalt für Wiederaufdau-KfW e o Instituto Brasileiro de Qualidade Nuclear-IBQN. Para a coleta de dados foram realizadas seis oficinas durante o mes de julho de 2004, nos próprios municipios, momento em que foi aplicado um questionário. As necessidades de qualificacáo estáo centradas em acóes programáticas, relativas as diferentes fases da vida - infancia, adolescencia, adulta e idosa-, como também a legislacáo e métodos e procedimentos comuns ao fazer do/a enfermeiro/a. Atentamos a necessidades de uma formacáo dos enfermeiros com referencia as concepcóes de promocáo da saúde, organizacáo do servico, cuidado familiar na perspectiva do conceito de territorio.

\section{ABSTRACT}

The study aims to identify the qualification needs and the nurse profiles in the Family Health Strategy (FHS). An exploratory-descriptive research was carried out with 36 male nurses in the municipal districts of Acaraú, Coreaú, Chaval, Granja, Marco, and Santana of the Acaraú, that work in the HSF, and students of the specialization course in residence in family health supplied by the Graduate School in Family Health, Visconde of Sabóia, Brazil. The municipal districts are part of the project Basic Actions of Health in Ceará, Brazil - Program of Qualification of the Secretary of Health of the State of Ceará in partnership with the German agency Kreditanstalt für Wiederaufdau-KfW and the Instituto Brasileiro de Qualidade Nuclear (IBQN). For the collection of data six workshops were carried out during July, 2004, in the municipal districts, at which time a questionnaire was completed. The qualification needs are centered in program actions, relative to the different phases of life -childhood, adolescence, adult and senior -, as well as common legislation, methods and procedures of nursing duties. We catered to the necessities of nurse training in regards to the concepts of promoting health, organizing services and family care in the concept of location..

\section{INTRODUCCIÓN}

En Brasil, en 1994, el Ministerio de Salud, como estrategia organizativa de la Atención Primaria de la Salud-APS, con la intención de cumplir los acuerdos pactados en Alma Ata en 1978, instituye el Programa Salud de la Familia-PSF. Este programa fue creado con el objetivo de "mejorar el estado de salud de la población a través de un modelo de asistencia, dirigido a la familia y a la comunidad, que incluya desde la protección y la promoción de la salud a la identificación precoz y el tratamiento de enfermedades". ${ }^{18}$

En 1997, el Ministerio de Salud admite que el PSF es una estrategia, por su alcance, su impacto y resolución, además de buscar la "[...] reversión del modelo asistencial vigente. Por eso, [...] su comprensión sólo es posible a través del cambio del objeto de atención, forma de actuación y organización general de los servicios, reorganizando la práctica asistencial en nuevas bases y criterios" $2: 8$, pasando entonces de la denominación de PSF para la Estrategia Salud de la Familia-ESF.

En la época de creación del PSF, como en la actualidad, la ESF exige una unidad de referencia, un equipo minimo compuesto por un enfermero, un médico, un auxiliar de enfermeria y de cuatro a seis Agentes Comunitarios de Salud, estos últimos viviendo en el territorio de actuación. Este equipo se responsabilizaría de 800 a 1.000 familias. La 
Enfermería, con el advenimiento de la ESF, está actuando en la búsqueda de la transformación del modelo propio del quehacer de la categoría, que se basaba en el concepto de salud como ausencia de enfermedad, centrado en la práctica de curación hospital-centro, herencia del sistema de salud vigente en el país durante décadas, para buscar en fin, emplear el concepto de salud como calidad de vida, con foco en la APS.

La ESF amplía el mercado de trabajo en enfermería e interioriza geográficamente enfermeros y auxiliares de enfermería, influyendo en el desarrollo del nuevo saber y prácticas, para además de las pragmáticas del cuidado de la cama hospitalaria de los individuos, descontextualizar sus vidas, necesidades, familias, culturas y lugar de domicilio.

Para eso, los enfermeros constantemente han buscado cualificarse con la intención de mejorar la calidad de su trabajo en la ESF, por medio de cursos de corta duración o de nivel de Especialización en Salud de la Familia o Salud Pública. A pesar de eso, todavía son muchas las fragilidades en el quehacer del enfermero, por una actuación competente en el cuidado a las familias, individuos, comunidades y grupos de riesgo o en situación de vulnerabilidad en la ESF.

Así, buscando cualificar mejor el proceso de trabajo del enfermero que actúa en la APS, la Escuela de Formación en Salud de la Familia Vizconde de Sabóia, por medio del Proyecto Acciones Básicas de Salud del Ceará - Programa de Cualificación de la Secretaria de Salud del Estado del Ceará - Brasil, en sociedad con la agencia alemana Kreditanstalt für Wiederaufdau-KfW y el Instituto Brasilero de Calidad Nuclear-IBCN, desarrolló esta investigación en el intento de identificar las necesidades de cualificación y el perfil de los enfermeros de la ESF.

\section{SUJETOS Y MÉTODO}

El estudio es de tipo exploratorio-descriptivo, realizado con 36 Enfermeros de las Provincias de Acaraú, Coreaú, Chaval, Granja, Marco e Santana do Acaraú, Estado do Ceará - Brasil, que actúan en la ESF y alumnos del Curso de Especialización en la modalidad de Residencia en Salud de la Familia, administrado por la Escuela de Formación en Salud de la Familia Vizconde de Sabóia. Las Provincias forman parte del Proyecto Acciones Básicas de Salud del Ceará - Programa de Cualificación de la Secretaria de Salud del Estado del Ceará en sociedad con la agencia alemana KfW y el IBCN.

Para la colecta de datos fueron realizadas seis oficinas durante el mes de julio de 2004 , en las propias provincias, momento en el que fue aplicado un cuestionario con variables referentes al sexo, edad, tiempo de egreso y de actuación en la ESF, que están expuestos en forma tabular; además de las necesidades de cualificación, que están presentadas en categorías.

En relación a las categorías, fue utilizado el referencial que Minayo sugiere para el establecimiento de clasificaciones. La autora complementa afirmando que trabajar con categorías significa "agrupar elementos, ideas o expresiones en torno de un concepto capaz de abarcar todo eso. Ese tipo de procedimiento, de un modo general, puede ser utilizado en cualquier tipo de análisis en pesquisa cualitativa". Minayo acaba refiriendo que las categorías "pueden ser establecidas antes del trabajo de campo, en la fase exploratoria de la investigación, o a partir de la colecta de datos". Las categorías "establecidas antes son conceptos más generales y más abstractos. Ese tipo requiere un fundamento teórico sólido por parte del investigador". 3:70 
La investigación tuvo el permiso de las Secretarias Municipales de Salud, como también de los sujetos del estudio, siendo aclarados los objetivos de la investigación, conforme la Resolución No. 196/1996 del Consejo Nacional de Salud- CNS, en que fueron respetados los principios básicos de la pesquisa involucrando seres humanos: la beneficencia, la justicia y no-maleficencia y la autonomía. ${ }^{4}$

\section{PRESENTACIÓN Y DISCUSIÓN DE LOS RESULTADOS}

\subsection{Perfil de los Enfermeros}

Tabla 1 Distribución de los enfermeros, según la edad, sexo y el tiempo de egreso y de actuación en la Estrategia Salud de la Familia. Ceará, Brasil, 2004.

\begin{tabular}{|c|c|c|}
\hline Variables & $N^{o}$ & $\%$ \\
\hline \multicolumn{3}{|l|}{ Edad } \\
\hline 23 a 26 & 12 & 33,3 \\
\hline 27 a 30 & 7 & 19,4 \\
\hline 31 a 34 & 3 & 8,4 \\
\hline 35 a 38 & 4 & 11,1 \\
\hline 39 a 42 & 4 & 11,1 \\
\hline 43 a más & 5 & 13,9 \\
\hline No respondió & 1 & 2,8 \\
\hline Total & 36 & 100,0 \\
\hline \multicolumn{3}{|l|}{ Sexo } \\
\hline Femenino & 30 & 83,3 \\
\hline Masculino & 6 & 16,7 \\
\hline Total & 36 & 100,0 \\
\hline \multicolumn{3}{|c|}{ Tiempo de Egreso (años) } \\
\hline$<1$ & 5 & 13,9 \\
\hline 1 a 2 & 5 & 13,9 \\
\hline 3 a 4 & 7 & 19,4 \\
\hline 5 a 6 & 6 & 16,7 \\
\hline 7 a 8 & 3 & 8,4 \\
\hline 9 a más & 10 & 27,7 \\
\hline Total & 36 & 100,0 \\
\hline \multicolumn{3}{|c|}{$\begin{array}{l}\text { Tiempo de Actuación en la ESF } \\
\text { (años) }\end{array}$} \\
\hline$<1$ & 8 & 22,2 \\
\hline 1 a 2 & 8 & 22,2 \\
\hline 3 a 4 & 9 & 25,0 \\
\hline 5 a 6 & 7 & 19,4 \\
\hline 7 a 8 & 2 & 5,6 \\
\hline 9 a 10 & 2 & 5,6 \\
\hline Total & 36 & 100,0 \\
\hline
\end{tabular}

En lo que concierne a las edades de los enfermeros, la mayoría se encuentra en el rango de edad de 23 a 30 años, $19(52,8 \%)$. En un estudio realizado en la provincia de Sobral Ceará en el año 2003 se muestra un perfil de los enfermeros de la ESF, con $33 \%$ de estos en un rango de edad de 22 a 29 años. ${ }^{5}$ 
En la actualidad la ESF está mostrándose como un campo floreciente de empleo para los enfermeros, médicos y odontólogos, siendo una puerta de entrada al mercado de trabajo y al Sistema Único de Salud - SUS por los egresos de los cursos universitarios de salud. Una situación contraria experimentan los enfermeros con mayor tiempo de egreso y servicio, que se encuentran en su mayoría concentrados en las organizaciones hospitalarias, por haber sido históricamente el escenario natural del trabajo de los mismos y consecuentemente, su mayor empleador.

En relación al sexo, $30(83,3 \%)$ de los enfermeros son del sexo femenino. Según las autoras, en "Brasil, la Enfermería es una acción, o una actividad, realizada predominantemente por mujeres que necesitan de eso para reproducir su propia existencia [ j" 6:95

De acuerdo con el Instituto de Estudios y Pesquisas - INEP del Ministerio de Educación MEC, en un estudio comparativo acerca de las matriculas de la enseñanza superior de graduación en el Brasil, los años de 1996 y 2003, demuestra un predominio creciente de mujeres, $54,4 \%$ y $56,4 \%$ respectivamente. En el caso de Enfermería, $84,7 \%$ de las matriculas el año de 2003 fueron del sexo femenino. ${ }^{7}$

El sexo femenino siempre ha predominado en la Enfermería Brasileña, como en la Enfermería mundial. En el caso de la Enfermería en Brasil, su formación, desde su génesis, tuvo como objetivo la preparación teórica y práctica, o sea, la formación de enfermeras.

La primera Escuela de Enfermería, la Escuela Profesional de Enfermeros y Enfermeras del Hospicio Nacional de Alienados - después Escuela de Enfermería Alfredo Pinto -, a pesar de la denominación también en masculino, tuvo la finalidad de preparar enfermeras para hospitales y hospicios militares y civiles. El Curso de Enfermería del Hospital Evangélico, actual Hospital Samaritano de Sao Paulo, tuvo la misma finalidad.

La Escuela de Enfermeras - actual Escuela de Enfermería Ana Néri - fue creada en 1923 por el Departamento Nacional de Salud Pública, para trabajar en pro de la mejora de las condiciones sanitarias de la población, tenía como objetivo hacer Enfermería. Esa escuela se considera la primera en trabajar en los moldes nightgalianos, referencia de la Enfermería Moderna $^{8}$, centrada en la actividad femenina. La historia de la Enfermería Brasileña justifica el motivo por el cual siempre hubo un predominio de mujeres en la Graduación en Enfermería.

En lo concerniente al origen de la Enfermería mundial, un autor relata que la misma está intrínsicamente asociada al cuidado materno. Con el paso del tiempo, el papel de la enfermería fue ampliándose, de manera gradual, superando la función biológica de la madre que cuida y amamanta a su hijo recién nacido, para significar su cuidado al enfermo, al anciano, a los necesitados y a los pobres. ${ }^{\circ}$

De los enfermeros, $17(47,2 \%)$ son egresados hace 4 años y $25(69,4 \%)$ ya están ejerciendo 4 años en la ESF. En la muestra existen enfermeros (dos - 5,6\%) que están ejerciendo en la ESF desde su establecimiento en el año 1994.

Un estudio realizado por Cavalcante y Ximenes Neto, en 2003, en la provincia de Sobral, reveló que $97 \%$ de los enfermeros eran del sexo femenino; $53 \%$ estaban en el rango de edad de 22 a 30 años, y $73,5 \%$ eran casados. ${ }^{10}$ La investigación titulada Perfil de los Médicos y Enfermeros del Programa Salud de la Familia en Brasil realizada en la 
Región Nordeste del Brasil el año 1999, muestra con relación a los enfermeros que 91,4\% eran del sexo femenino, 38,6\% estaban en el rango de edad de hasta 29 años y $42 \%$ entre 30 a 39 años, $45,3 \%$ con hasta cuatro años de egreso, y 41,1\% estaba hace menos de un año actuando en el PSF."

\subsection{Necesidades de Cualificación de los Enfermeros de la ESF}

Las referencias hechas por los entrevistados fueron agrupadas para un mejor análisis de los datos, y sistematizadas a partir de las siguientes subcategorías: Cuidados de enfermería de la salud del niño; Cuidados de enfermería de la salud del adolescente; Cuidados de enfermería de la salud del adulto; Cuidados de enfermería de la salud del anciano; Organización del servicio.

\subsubsection{Cuidados de enfermería de la salud del niño}

- Visita domiciliaria al recién nacido

- Puericultura/promoción de la salud del niño: evaluación del crecimiento y del desarrollo, alimentación, vacunación, higienización del bebé

- Consulta de acompañamiento al niño de cero a nueve años

- Examen físico del niño

- Metodología de la asistencia en enfermería

- Atención integrada a las Enfermedades Prevalecientes en la InfanciaAIDPI

- Situaciones de vulnerabilidad de la salud del niño: accidentes y violencias

- Abordaje al niño saludable

- Abordaje al niño con necesidades especiales

- Acciones de educación en salud.

Las necesidades de cualificación acerca del cuidado de enfermería al niño, conforman temas relacionados con la promoción y educación en salud, prevención de enfermedades y cuidados domiciliarios. En realidad, las necesidades comprenden las acciones básicas para el desarrollo del cuidado integral al niño.

\subsubsection{Cuidados de enfermería de la salud del adolescente}

- Trabajo con grupos;

- Consulta al adolescente

- Examen físico

- Metodología de la asistencia de enfermería

- Situaciones de vulnerabilidad en la salud del adolescente

- Abordaje al adolescente saludable

- Técnicas de comunicación y aspectos éticos

- Acciones de promoción y educación en salud.

Ante la semejanza de las necesidades de cualificación sobre la atención de enfermería de la salud del niño con la salud del adolescente, son presentados temas básicos, relativos al cuidado integral de enfermería de los referidos sujetos, así como las técnicas de buena comunicación verbal y principios éticos básicos, relativos al cuidado 
integral de enfermería de los referidos sujetos, y también, las técnicas de buena comunicación verbal y principios éticos a seguir durante la consulta de enfermería.

\subsubsection{Cuidados de enfermería de la salud de adultos}

- Atención al portador de diabetes mellitus, hipertensión arterial, tuberculosis y lepra

- Atención a las enfermedades relacionadas con el trabajo

- Acciones de promoción y educación de salud

- Verificación de señales vitales y medidas antropométricas

- Examen físico

- Metodología de asistencia y diagnóstico de enfermería

- Salud de la Mujer:

- Prevención del cáncer uterino-cervical y de mama con organización del servicio

- Humanización del prenatal y puerperio y trabajo con grupos de gestantes

- Atención del climaterio

- Situaciones de vulnerabilidad de la salud de la mujer

- Abordaje sindromático de la Enfermedades Sexualmente TransmisiblesEST

- Métodos contraceptivos.

Las necesidades de cualificación en los cuidados de enfermería de la salud de adultos se refieren al proceso de salud-enfermedad de la comunidad, habiendo una predominancia del cuidado en una perspectiva de curación de las enfermedades crónicas e infectocontagiosas comunes de este rango de edad y del perfil epidemiológico de la región, además del cuidado integral de la mujer adulta en la perspectiva de la salud sexual y reproductiva.

\subsubsection{Cuidados de enfermería de la salud del anciano}

- Atención domiciliaria al anciano encamado

- Situaciones de vulnerabilidad de la salud del anciano

- Acciones de promoción y educación en salud

- Trabajo con grupos

- Técnica - sonda nasogástrica y vesical.

Las necesidades de cualificación para un competente cuidado de enfermería al anciano comprenden prácticas asistenciales de las enfermedades que producen impedimentos, la promoción de salud, además de técnicas instrumentales básicas.

\subsubsection{Organización del servicio}

- Desarrollo de liderazgo en Enfermería

- Legislación de Enfermería

- Organización del Servicio de Archivo Familiar y Estadística-SAFE (informatización) y prontuario familiar y actualización de las fichas de las familias 
- Limpieza de la Unidad Básica de Salud-UBS y destino de las deposiciones/colecta selectiva de residuos biológicos, químicos, radioactivos e comunes

- Abordaje externo de la UBS - higienización

- Organización de la sala de aerosolterapia

- Organización de la sala de curativos y cuidado de heridas

- Organización de la sala de Terapia de Rehidratación Oral- TRO

- Organización de la sala de vacunación

- Rutinas de vacunación

- Técnicas de esterilización y desinfección del material

- Bioseguridad

- Organización y estructuración de materiales y equipos

- Suministro de insumos

- Organización de los consultorios

- Acogimiento y recepción de la UBS

- Organización de la reunión con el equipo de enfermería

- Elaboración de normas y rutinas de la UBS

- Colecta y transporte de material para exámenes de laboratorio

- Implementación de la organización de la farmacia

- Primeros socorros en la comunidad.

Las necesidades de cualificación en la organización del servicio abarcan acciones y servicios de la UBS, técnicas instrumentales básicas de enfermería, además de estrategias de liderazgo. Se nota que los enfermeros no apuntan necesidades acerca de la gerencia del área adscrita a la UBS, tal vez debido al hecho de que el proceso de trabajo de los mismos está centrado, predominantemente, en los espacios internos de la unidad de salud.

\section{CONCLUSIONES}

De los 36 enfermeros entrevistados, la mayor parte eran mujeres en el rango de edad de 23 a 30 años, tienen hasta 4 años de egreso y 4 años de actuación en ESF. Las necesidades de cualificación adoptadas por ellos fueron clasificadas en: Cuidados de enfermería de la salud del niño; Cuidados de enfermería de la salud del adolescente; Cuidados de enfermería de salud de adultos; Cuidados de enfermería de la salud de ancianos; y Organización del servicio. La mayor demanda fue puntualizada en el área de la organización del servicio.

\section{CONSIDERACIONES FINALES}

El estudio apunta que las necesidades de cualificación de los enfermeros están centradas en el modelo de atención de la salud basado en Acciones Programáticas de Salud, que utiliza la "programación como instrumento de redefinición del proceso de trabajo en salud, tomando como punto de partida la identificación de las necesidades sociales de salud de la población que demanda los servicios de las UBS" y en el modelo de la ESF, que incluye "acciones educativas y de prevención de riesgos y agravios específicos, con acciones básicas de atención de la salud de grupos prioritarios", ${ }^{2165}$ además de acciones de promoción de la salud en una perspectiva de salud con calidad de vida y desarrollo del cuidado integral, holístico, humanizado hacia las familias, sujetos y comunidades.

En cuanto a la educación de los profesionales de salud, la OMS afirma que ha sido ampliamente criticada por no estar dirigida a las necesidades de salud de la sociedad. La 
mayoría de los profesionales tienen una formación densa en el campo biomédico y clínico pero una corta formación en Ciencias Sociales y Humanas, lo que reduce sus posibilidades de manejar cuestiones políticas, sociales, culturales y económicas, exigidas por la gerencia. Son escasas las oportunidades de aprender cómo orientar a los poderes sociales, económicos y políticos que interfieren en el sector de la salud.

El texto de la OMS afirma además que las instituciones responsables de la capacitación de los profesionales de salud están alejadas de los espacios de la toma de decisiones, acerca de la provisión de servicios y de las políticas de salud.13

Es sabido que la cualificación de los trabajadores de salud, principalmente los de la ESF, es de fundamental necesidad debido a los avances teóricos, organizacionales, tecnológicos y políticos ocurridos y la diversidad, tanto en el campo de la atención, como en la gestión en el territorio, lo que impone diariamente nuevas situaciones a afrontar. La Educación Permanente en Salud permite el re-significado del proceso de trabajo, por ser desarrollada su práctica en el servicio y la apropiación afectiva del territorio, con sus necesidades de salud sentidas o no. ${ }^{14}$

Según los Ministerios de Salud y de Educación del Brasil, "los procesos formativos y las prácticas profesionales en salud demuestran compromiso con diversos intereses, pero muchas veces excluyen los de los usuarios del SUS.

En general, el panorama que observamos en el área de la Salud, sea a nivel federal, estatal o municipal, sea en la gestión, en la enseñanza o en los servicios, perpetúa modelos conservadores y parece distanciado de un modelo lógico, que sería el "usuariocentrado" y de la primera misión que seria un cuidado adecuado a las necesidades de la población". 15:11

Conforme Carvalho e Ceccim, "para ser un profesional de salud existe la necesidad de conocimiento científico y tecnológico, pero también de conocimiento de naturaleza humanística y social relativo al proceso de cuidar, de desarrollar proyectos terapéuticos singulares, de formular y evaluar políticas y de coordinar y conducir sistemas y servicios de salud. El diploma en cualquier área de salud no es suficiente para garantizar la cualificación necesaria, ya que el conocimiento y la información están en permanente cambio y exigen actualización del profesional $[\ldots . .]^{15}$

El quehacer del enfermero en la ESF comprende una diversidad de actividades y responsabilidades que van desde el cuidado de los sujetos, familias y comunidades en las diferentes fases de la vida, en la organización del servicio propio de las prácticas cotidianas de Enfermería, además de cada vez más agregar la gerencia del territorio de la ESF.

En realidad, la actuación en la ESF exige una diversidad del saber y prácticas en las áreas relacionadas con la gestión sanitaria, el cuidado de las familias, sujetos y poblaciones, el manejo de las determinaciones y consecuencias sociales, del territorio de actuación, de las concepciones y prácticas en salud. Tales conocimientos, muchas veces, no son ofrecidos durante la educación universitaria, conduciendo a los profesionales a especializarse en varias áreas para lograr un proceso de trabajo vastísimo en ESF.

Se constituye en un desafío para los gestores públicos y para las instituciones educadoras de Enfermería en el país ofrecer respuestas a esas necesidades apuntadas, en el sentido de cualificar mejor al enfermero que actúa en esta estrategia, cuya perspectiva debe ser ampliada cada vez más. 


\section{REFERENCIAS}

1. Ministerio da Saúde (BR). Fundagáo Nacional de Saúde. Departamento de Operagöes. Coordenagáo de Saúde da Comunidade. Programa Saúde da Familia: saúde dentro de casa. Brasilia: Ministerio da Saúde; 1994.

2. Ministerio da Saúde $(B R)$. Secretaria de Assistència à Saúde. Coordenagáo de Saúde da Comunidade. Saúde da Familia: uma estrategia para a reorientagáo do modelo assistencial. Brasilia. Ministerio da Saúde; 1997. 36p.

3. Minayo MCS. Ciencia, técnica e arte: o desafio da pesquisa social. In: Minayo MCS, organizadora. Pesquisa social: teoria, método e criatividade. 22 ed. Petrópolis-RJ: Vozes; 2003. p. 9-29.

4. Ministerio da Saúde (BR). Fundagáo Nacional de Saúde. Diretrizes e normas reguladoras de pesquisas. Informe epidemiológico do SUS 1996; 5(2, supl. 3):1-14.

5. Figueiredo MAC, Ximenes Neto FRG, Andrade LOM. Assistència à clientela portadora de hanseniase na Atengáo Primària à Saúde: uma investigagáo das agöes realizadas por enfermeiros. Enfermagem Atual 2005; 5(29):19-23.

6. Chiesa AM, Fracolli LA, Sousa MF. Enfermeiros capacitados para atuar no Programa Saúde da Familia na cidade de Sao Paulo: relato de uma experiencia. Saúde em Debate 2004; 28:89-90.

7. Godinho T, organizador. Trajetória da mulher na educagáo brasileira 1996-2003. Brasilia: Instituto Nacional de Estudos e Pesquisas Educacionais Anisio Teixeira; 2006.

8. Fernandes JD. A trajetória do ensino de graduagáo em enfermagem no Brasil. In: Teixeira E, Vale EG, Fernandes JD, Sordi MRL, organizadores. O ensino de graduagáo em enfermagem no Brasil: o ontem, o hoje e o amanhá. Brasilia: Instituto Nacional de Estudos e Pesquisas Educacionais Anisio Teixeira-INEP; 2006. p. 9-21.

9. Lunardi VL. História da Enfermagem: rupturas e continuidades. 2a ed. Pelotas: Editora do Autor; 2004.

10. Cavalcante JHV, Ximenes Neto FRG. O adolescente na atengáo primària em saúde: uma análise das agöes realizadas por Enfermeiros na Estrategia Saúde da Familia. Rev. Paulista de Enfermagem 2004; 23(3-4):242-7.

11. Machado $\mathrm{MH}$, coordenadora. Perfil dos Médicos e Enfermeiros do Programa Saúde da Familia no Brasil: relatório final - Brasil e Grandes Regiöes. Brasilia: Ministerio da Saúde; 2000. v. I.

12. Paim JS. Politicas de Saúde no Brasil. In: Rouquayrol MZ, Almeida Filho N. Epidemiologia \& Saúde. 6a ed. Rio de Janeiro: MEDSI; 2003. Cap. 20. p. 587-604.

13. Organización Mundial de la Salud-OMS. Una formación profesional mas adecuada del personal sanitario: Informe de un grupo de estudio de la OMS sobre la enseñanza orientada a la solución de problemas en las profesiones sanitarias. Ginebra: OMS; 1993. (OMS, Serie de informes técnicos; 838).

14. Ximenes Neto FRG. Gerenciamento do território na Estratégia Saúde da Familia: o processo de trabalho dos gerentes. 2007. 463 f. Dissertagáo (Mestrado Académico em Saúde Pública), - Universidade Estadual do Cearà-UECE, Fortaleza; 2007.

15. Ministerio da Saúde (BR). Ministerio da Educagáo. A aderéncia dos cursos de graduagáo em enfermagem, medicina e odontologia às diretrizes curriculares nacionais. Brasilia: Ministerio da Saúde; 2006. 162 p.: il. - (Série F. Comunicagáo e Educagáo em Saúde).

16. Carvalho YM, Ceccim RB. Formagáo e educagáo em saúde: aprendizados com a saúde coletiva. In: Campos GWS, colaboradores. Tratado de Saúde Coletiva. 1. reimp. Sao Paulo: HUCITEC; Rio de Janeiro: FIOCRUZ; 2007. p. 137-170. 
